\title{
ON THE GENERAL TERM IN THE REVERSION OF SERIES.
}

BY PROF. JAMES MOMAHON.

IN reverting the series

$$
y=a_{0} x+a_{1} x^{2}+a_{2} x^{3}+\ldots \quad\left[a_{0} \neq 0\right]
$$

it is usual to assume a development for $x$ in the form

$$
x=A_{0} y+A_{1} y^{2}+A_{2} y^{8}+\ldots,
$$

and then to substitute, and equate coefficients of like powers, thus determining $A_{0}, A_{1}, \ldots$ in succession.

This method does not give any observable law for the independent formation of the expression for the coefficient of a given power of $y$.

A different method, however, based on Lagrange's series, furnishes the desired general term.

The first equation may be written

$$
a_{0} x=y-a_{1} x^{2}-a_{2} x^{3}-\ldots,
$$

or

$$
x=z+b_{1} x^{2}+b_{2} x^{3}+\ldots,=z+\phi(x),
$$

where

$$
z=\frac{y}{a_{0}}, \quad b_{1}=-\frac{a_{1}}{a_{0}}, \quad b_{2}=-\frac{a_{2}}{a_{0}}, \ldots
$$

and

$$
\phi(x)=b_{1} x^{2}+b_{2} x^{3}+\ldots ;
$$

whence, by Lagrange's series,

$$
x=z+\phi(z)+\frac{1}{2 !} \frac{d}{d z}[\phi(z)]^{2}+\frac{1}{3 !} \frac{d^{2}}{d z^{2}}[\phi(z)]^{3}+\ldots
$$


1894] GENERAL TERM IN REVERSION OF SERIES.

in which

$$
\begin{aligned}
& \phi(z)=b_{1} z^{2}+b_{2} z^{3}+\ldots . \\
& \frac{d}{d z}[\phi(z)]^{2}=4 b_{1}{ }^{2} z^{3}+5\left(2 b_{1} b_{2}\right) z^{4}+6\left(b_{2}{ }^{2}+2 b_{1} b_{3}\right) z^{3}+\ldots, \\
& \frac{d^{2}}{d z^{2}}[\phi(z)]^{3}=6.5 b_{1}{ }^{3} z^{4}+7.6\left(3 b_{1}{ }^{2} b_{2}\right) z^{5} \\
& +8 \cdot 7\left(3 b_{1} b_{2}{ }^{2}+3 b_{1}{ }^{2} b_{3}\right) z^{0}+\ldots ., \\
& \therefore x=z+b_{1} z^{2}+\left(b_{2}+\frac{4}{2 !} b_{1}{ }^{2}\right) z^{3}+\left(b_{3}+\frac{5}{2 !} \cdot 2 b_{1} b_{2}+\frac{5.6}{3 !} b_{1}{ }^{8}\right) z^{4} \\
& +\left[b_{4}+\frac{6}{2 !}\left(b_{2}{ }^{2}+2 b_{1} b_{3}\right)+\frac{6 \cdot 7}{3 !} \cdot 3 b_{1}{ }^{2} b_{2}+\frac{6 \cdot 7 \cdot 8}{4 !} b_{1}{ }^{4}\right] z^{5} \\
& + \text {. } . \\
& +\left\lceil b_{n-2}+\frac{n}{2 !}\left(2 b_{1} b_{n-3}+2 b_{2} b_{n-4}+\ldots\right)\right. \\
& +\frac{n^{2 \mid 1}}{3 !}\left(3 b_{1}{ }^{2} b_{n-4}+6 b_{1} b_{2} b_{n-4}+\ldots\right) \\
& \left.+\frac{n^{3 \mid 1}}{4 !}\left(4 b_{1}{ }^{3} b_{n-5}+12 b_{1}{ }^{2} b_{2} b_{n-6}+\ldots\right)+\ldots\right] z^{n-1} \\
& + \text {. } . \quad . \quad \text {; }
\end{aligned}
$$

wherein

$$
n^{r \mid 1}=n(n+1)(n+2) \ldots(n+r-1),{ }^{*}
$$

and the parenthesis that $\frac{n^{r \mid 1}}{(r+1) !}$ multiplies, is

in which

$$
\Sigma^{(p+q+\ldots) !} b_{i}{ }^{p} b_{j}^{q} \ldots,
$$

$$
p+q+\ldots=r+1, \text { and } p i+q j+\ldots=n-2
$$

* In accordance with the notation

$$
n^{r \mid d}=n(n+d)(n+2 d) \ldots[n+(r-1) d] .
$$


that is to say, the order of this parenthesis in the letters $b_{1}, b_{2}, \ldots$ is $r+1$, its weight is $n-2$, and the numerical coefficients are those of the polynomial theorem.

In terms of the original letters $y, a_{0}, a_{1}, \ldots$ the series may be written in the more homogeneous form

$$
\begin{aligned}
& x=\frac{y}{a_{0}}+\frac{y^{2}}{a_{0}{ }^{3}}\left(-a_{1}\right)+\frac{y^{3}}{a_{0}{ }^{6}}\left(-a_{0} a_{2}+\frac{4}{2 !} a_{1}{ }^{2}\right) \\
& +\frac{y^{4}}{a_{0}{ }^{7}}\left(-a_{0}{ }^{2} a_{3}+\frac{5}{2 !} \cdot 2 a_{0} a_{2} a_{2}-\frac{5^{2 \mid 1}}{3 !} a_{1}{ }^{3}\right) \\
& +\frac{y^{6}}{a_{0}^{0}}\left[-a_{0}^{3} a_{4}+\frac{6}{2 !} a_{0}{ }^{2}\left(a_{2}{ }^{2}+2 a_{1} a_{3}\right)-\frac{6^{2 \mid 1}}{3 !} a_{0}\left(3 a_{1}{ }^{2} a_{2}\right)+\frac{6^{311}}{4 !} a_{1}^{4}\right] \\
& +\cdot \cdot \cdot \\
& +\frac{y^{n-1}}{a_{0}^{2 n-3}}\left[-a_{0}{ }^{n-3} a_{n-2}+\frac{n}{2 !} a_{0}{ }^{n-4}\left(2 a_{3} a_{n-3}+\ldots\right)\right. \\
& \left.-\frac{n^{2 \mid 1}}{3 !} a_{0}{ }^{n-5}\left(3 a_{1}{ }^{2} a_{n-4}+\ldots\right)+\ldots+\frac{n^{n-31}}{(n-2) !}\left(-a_{1}\right)^{n-2}\right] \\
& + \text {. . . . }
\end{aligned}
$$

It will be noticed that the coefficient of $\frac{y^{n-1}}{a_{0}^{2 n-3}}$ is now a homogeneous function of $a_{0}, a_{1} \ldots$, of order $n-2$, weight $n-2$; and that the "polynomial coefficients" involved in such terms as $\frac{n^{3 \mid 1}}{4 !} a_{0}{ }^{n-6}\left(12 a_{1}{ }^{2} a_{2} a_{n-6}+\ldots\right)$ are to be chosen without reference to the exponent of $a_{0}$; while the latter exponent is related to the outside coefficient $\frac{n^{3 \mid 1}}{4 !}$, by an obvious rule.

If $a_{0}=0$, let $a_{m-1} x^{m}$ be the first term in the given series, then the relation between $x$ and $y$ may be written in the form $x=f[z+\phi(x)]$, where $z=\frac{y}{a_{m-1}}$, and $f(z)=z^{\frac{1}{m}}$; hence Laplace's theorem gives a development for $x$ that can be arranged in powers of $z^{\frac{1}{m}}$. As the general term now involves both $n$ and $m$, the law is not so simple as in the case above, for which $m=1$. 